\title{
Des lasers sans inversion de population
}

M.-C. Castex

Laboratoire de Physique des Lasers, Université Paris-Nord, 93430 Villetaneuse, France

Les lasers sans inversion de population (SIP) constituent un sujet d'étude en pleine expansion non seulement sur le plan théorique mais aussi expérimental (1-6). Des expériences récentes confirment la possibilité d'un effet de gain dans un système a 4 niveaux qui ne présente pas d'inversion de population apparente, celle-ci peut être "cachée" (3).

L'amplification SIP peut être réalisée soit en réduisant 1'absorption grâce à la préparation d'un piégeage cohérent des sous-niveaux de l'état fondamental, soit en utilisant des effets d'interférences quantiques qui permettent de briser la symétrie entre le profil d'émission stimulée et l'absorption. En particulier, dans un système atomique à 3 niveaux, une transparence peut être induite (EIT) par l'application d'un champ laser externe en résonance sur deux niveaux ou encore un continuum d'ionisation peut être structuré (LICS) en introduisant un couplage radiatif entre un état lié et le continuum de l'atome.

Les avantages présentés par ces systèmes simples seront discutés. Mais des schémas de plus en plus complexes sont actuellement proposés. On tentera de donner ici un aperçu de l'état de l'art en précisant les conditions d'observation du phénomène SIP.

(1) O. Kocharovskaya, Physics. Reports 219, 175 (92)

(2) M.O Scully, Physics Reports 219191 (92)

(3) W.E. van der Veer, R.J.J van Diest, A. Donszelmann and H.B. van Linden van den Heuvell, Phys. Rev. Lett 703243 (93)

(4) A. Nottelmann, C. Peters and W. Lange, Phys. Rev. Lett, 701783 (93)

(5) J.Gao, C.Guo, X.Guo, G. Jin, P. Wang, J. Zhao, H. Zhang, Y. Jiang, D. Wang and D. Jiang, Opt. Com 93323 (92)

(6) E.S. Fry, X.Li, D. Nikonov, G.G. Padmabandu, M.O. Scully, A.V. Smith, F.K.Tittel, C.Wang, S.R.Wilkinson and S.Y.Zhu, Phys. Rev. Lett 703235 (93) 